University of Miami, FL. Mean age was 34 years (range, 2-87 years). Valproate aqueous solution $(500 \mathrm{mg} / 5 \mathrm{ml}$ ), one-fourth daily dose (median dose $375 \mathrm{mg}$ or $5.1 \mathrm{mg} / \mathrm{kg}$ ), diluted with $50 \mathrm{ml}$ normal saline or $5 \%$ dextrose/water, infused over 1 hour, repeated 6 hourly for up to 2 day's. Transient severe side effects in $54(17 \%)$ included headache, reaction at injection site, nausea, vomiting, somnolence ( $2 \%$ each), dizziness, and abnormal taste ( $1 \%$ each). Six left the study prematurely due to valproate intolerance: pain at IV site, amylase elevations, headache, nausea and vomiting. Abnormal serum chemistries following treatment in 7 generally returned to normal. (Devinsky $O$ et al. Safety of intravenous valproate. Ann Neurol Oct 1995;38:670-674). (Respond: Dr Devinsky, Department of Neurology, Hospital for Joint Diseases, 301 East 17 th Street, New York, NY 10003).

COMMENT. This study demonstrates the relative safety of IV valproate, which is not yet available for general use in the US. Previous studies in Europe, where the IV preparation is available, have demonstrated efficacy in neonatal seizures, and in neurosurgical adult patients with status epilepticus resistant to diazepam. The authors recommend further trials to determine optimal dose, efficacy, and safety.

\title{
LORAZEPAM V DIAZEPAM IN STATUS EPILEPTICUS
}

A prospective, open, odd and even dates trial of lorazepam compared to diazepam for the treatment of acute convulsions and status epilepticus in 102 children is reported from the Royal Liverpool Children's NHS Trust, UK. Lorazepam $(0.05-0.1 \mathrm{mg} / \mathrm{kg})$ and diazepam $(0.3-0.4 \mathrm{mg} / \mathrm{kg})$ controlled convulsions within 20 to 60 seconds in $76 \%$ and $51 \%$ of patients, respectively, after a single dose administered IV over 15 to 30 seconds. Multiple doses as well as additional AEDs were required in 17 patients who received an initial injection of diazepam compared to only 1 who received lorazepam. Respiratory depression occurred in 7 diazepam treated patients and necessitated admission to intensive care. No patient receiving lorazepam required intensive care. Rectal administration, when venous injection was not possible, was $100 \%$ effective with a single dose of lorazepam in 6 patients treated, whereas 13 of 19 patients receiving diazepam rectally required multiple doses, 12 required additional AEDs, 1 had respiratory depression, 2 were admitted to intensive care, and 7 relapsed with recurrence of seizures within 24 hours. (Appleton R et al. Lorazepam versus diazepam in the acute treatment of epileptic seizures and status epilepticus. Dev Med Child Neurol 1995;37:682-688). (Respond: Dr Richard Appleton, Royal Liverpool Children's NHS Trust, Alder Hey, Eaton Road, Liverpool L12 2AP, UK).

COMMENT. Lorazepam appears to be safe, at least as effective as diazepam in the initial control of acute convulsions, including status epilepticus, and more effective in sustaining seizure control. Rectal lorazepam was useful in infants when intravenous injection was impractical. Lorazepam has a longer half life than diazepam, and its duration of action is more prolonged, accounting for the more sustained control.

(See Progress in Pediatric Neurology I, 1991, PNB Publishers, pp124-5).

\section{RISK OF STEVENS-JOHNSON SYNDROME WITH AEDS}

An international case-controlled study of medication use and the risk of Stevens-Johnson syndrome or toxic epidermal necrolysis is reported by the Groupe Epidemiologie LY Stevens Johnson (ELYS), Department of Dermatology, 
and Department of Public Health, Universite Paris XII, Creteil, France; and centers in Boston, US; Milan, Italy; Freiburg, Germany; Porto, Portugal; and Toronto, Canada. In 245 patients hospitalized for treatment, the relative risks with various drugs were as follows: carbamazepine 90, phenytoin 53, phenobarbital 45, valproic acid 25, compared to 172 for sulfonamide antibiotics, 6.7 for aminopenicillins, and 54 for corticosteroids. For many other drugs in common use, including contraceptive pills, benzodiazepines, and phenothiazines, the risk of serious skin reactions was not increased. (Roujeau JC et al. Medication use and the risk of Stevens-Johnson syndrome or toxic epidermal necrolysis. N Engl I Med Dec 14, 1995;333:1600-7). (Reprints: Dr Roujeau, Service de Dermatologie, Hopital H Mondor, 94010 Creteil, France).

COMMENT. The incidence of toxic epidermal necrolysis is estimated at 0.4 to 1.2 cases per million person-years and of Stevens-Johnson syndrome, at 1 to 6 cases per million person-years. None of the above drugs caused an excess risk greater than 5 cases per million users per week. The excess risks ranged from a low of 0.2 per million for aminopenicillins to a high of 4.5 per million for sulphonamides. For the anticonvulsants, excess risks of these skin reactions ranged from a low of 0.7 for valproic acid to a high of 2.5 for carbamazepine. Despite the relatively low incidence, these skin syndromes may kill or lead to prolonged hospitalization and extreme discomfort. Patients introduced to any of the above anticonvulsants should be warned of the dangers of skin rash, especially within the first two weeks of treatment, and instructed to discontinue medication and report to a physician immediately at the first sign of reaction. Carbamazepine appears to be the worst offender, and valproic acid is not immune. Corticosteroids, a controversial treatment for Stevens-Johnson syndrome, carries a surprisingly increased risk of inducing the disorder. Benzodiazepines, having no excess risk of Stevens-Johnson syndrome, are the obvious agents to substitute when other anticonvulsants are discontinued due to these severe skin reactions.

Carbamazepine-induced skin rash is reviewed in Progress in Pediatric Neurology II, 1994, PNB Publishers, pp 107-109. A personal communication from Ciba-Geigy recorded 30 cases of carbamazepineinduced Stevens-Johnson syndrome, 8 erythema multiforme, and 5 toxic epidermal necrolysis (Lyell's syndrome) reported to the company in an eight year period, 1982-89. The above international study found 13 cases related to carbamazepine, and accounting for $5 \%$ of the total drug-

induced severe cutaneous reactions, during a four year period, 1989-93.

\section{MOVEMENT DISORDERS}

\section{COMORBID TOURETTE'S AND BIPOLAR DISORDERS}

Of 205 patients with Tourette's disorder in the North Dakota Longitudinal Tourette Syndrome Surveillance Project, 15 had comorbid bipolar disorder. The ratio of males to females was 5.2:1. The estimated risk of developing bipolar disorder among the study group of children and adolescents with Tourette's disorder was more than four times higher than the level expected by chance, significant at the 0.05 level. Males were at greater risk than females, and adults had comorbid developmental disorders as well. Shared common neural pathways, especially basal ganglia structures, and genetic factors may explain the comorbidity. (Kerbeshian J et al. Comorbid Tourette's disorder and bipolar 